\title{
Informe de un caso de infección por coronavirus en un trasplantado renal
}

\author{
Sara Mencía Prendes, María Ruiz García \\ Servicio de Nefrología. Hospital General Universitario Gregorio Marañón. Madrid. España
}

Como citar este artículo:

Mencía-Prendes S, Ruiz-García M. Informe de un caso de infección por coronavirus en un trasplantado renal.

Enferm Nefrol. 2020 Jul-Sep;23(3):294-302

\section{Resumen}

Introducción: La infección por coronavirus favorece el desarrollo de alteraciones respiratorias. En trasplantados renales el pronóstico de la neumonía por SARS-CoV-2 puede ser diferente al resto de la población. Muchos pacientes trasplantados tienen de base linfopenia inducida por fármacos, por lo que la detección y el tratamiento precoz son determinantes en este grupo de población.

Presentación del caso: Varón de 50 años con ERC no filiada, trasplantado renal en 2016 que acudió a urgencias por fiebre de $38^{\circ} \mathrm{C}$, tos, rinorrea, diarrea con pérdida de peso y molestia en injerto renal en fosa iliaca derecha. Se diagnosticó de COVID-19 positivo tras realización de placa de tórax y PCR SARS-CoV-2. Ingresó en nuestra unidad de trasplante renal.

Plan de cuidados: Se realizó una valoración inicial mediante los patrones funcionales de Gordon. Posteriormente identificamos Diagnósticos de Enfermería según la taxonomía NANDA con sus correspondientes criterios de resultados e intervenciones.

Evaluación del plan: Tras la realización de las intervenciones y la evaluación de los indicadores de resultados observamos una disminución de la temperatura corporal y de la dificultad respiratoria con mejoría en el patrón respiratorio. En cuanto a la función renal, no se ha visto significativamente alterada a pesar de la retirada temporal de la inmunosupresión.

\section{Correspondencia:}

María Ruiz García

Email: maguie_224@hotmail.com
Conclusión: El paciente se fue de alta con una función renal similar a la previa y con la inmunosupresión reintroducida. Se lograron los objetivos planteados dejando patente que el papel de enfermería ha sido fundamental en el proceso de recuperación y afrontamiento de la enfermedad.

PALABRAS CLAVE: coronavirus; inmunosupresores; trasplante renal; rechazo agudo.

\section{Report of a case of coronavirus infection in a renal transplant recipient.}

\begin{abstract}
Introduction: Coronavirus infection favours the development of respiratory disorders. In kidney transplant patients, the prognosis of SARS-CoV-2 pneumonia may be different from the rest of the population. Many transplant patients have drug-induced lymphopenia, so early detection and treatment are crucial in this population group.
\end{abstract}

Case description: A 50-year-old man with unknown CKD and a kidney transplant in 2016 who came to the emergency room due to fever of $38^{\circ} \mathrm{C}$, cough, rhinorrhea, diarrhea with weight loss and discomfort in a kidney graft in the right iliac fossa. He was diagnosed with COVID-19 positive after performing a chest X-ray and SARS-CoV-2 PCR. He was admitted to our kidney transplant unit.

Description of the care plan: An initial assessment was made using Gordon's functional patterns. Subsequently, 
we identify Nursing Diagnoses according to the NANDA taxonomy with their corresponding outcome and intervention criteria.

Evaluation of the care plan: After carrying out the interventions and evaluating the outcome indicators, we observed a decrease in body temperature and respiratory distress with improvement in the respiratory pattern. Regarding renal function, it has not been significantly altered despite the temporary withdrawal of immunosuppression.

Conclusion: The patient is discharged with a renal function similar to the previous one and with reintroduced immunosuppression. The objectives set were achieved, making it clear that the role of nursing has been vital in the process of recovery and coping with the disease.

KEYWORDS: Coronavirus; immunosuppressants; kidney transplantation; acute rejection.

\section{Introducción}

España se ha visto muy afectada por la enfermedad COVID-191 producida por un virus RNA betacoronavirus que provoca el síndrome respiratorio agudo severo tipo 2 (SARS-CoV-2). Esta enfermedad es fácilmente transmisible por vía aérea con más de 300.000 casos positivos, 28.498 muertes confirmadas ${ }^{2}$ y alrededor de 44.000 muertes en exceso, hasta el 4 de agosto de $2020^{3}$. Recientes estudios han mostrado la presencia de una carga viral elevada en la orofaringe en etapas tempranas de la enfermedad, lo que aumenta la preocupación por alto riesgo de inefectividad durante los periodos de incubación o de leve sintomatología 4 . Se ha estimado un periodo de incubación de 5-6 días, con un rango de 1 a 14 días. No hay un tratamiento específico, como principal medida terapéutica se realiza el control sintomático de la enfermedad. Los síntomas de la enfermedad, en más de un $80 \%$ de los casos, son leves como fiebre, tos, expectoración, malestar general; mientras que un $20 \%$ pueden tener manifestaciones graves como neumonía severa, edema pulmonar, síndrome de dificultad respiratoria aguda o insuficiencia orgánica múltiple requiriendo ingreso hospitalario ${ }^{5,6}$.

Paciente con patologías previas, ancianos, inmunodeprimidos, mujeres embarazadas son más susceptibles de la COVID-19, pudiendo llegar a requerir atención en una unidad de cuidados intensivos ${ }^{7}$.
Los pacientes en tratamiento con diálisis y los receptores de trasplante renal constituyen un grupo de riesgo y presentan connotaciones relevantes desde el punto de vista epidemiológico ${ }^{8}$. En los trasplantados renales y debido al tratamiento con inmunosupresores, tanto las manifestaciones como el pronóstico y el tratamiento de la neumonía por SARS-CoV-2 pueden ser diferentes de los del resto de la población ${ }^{8}$. En la población general, se ha observado linfopenia hasta en el $63 \%$ de los pacientes ${ }^{9}$, pero muchos trasplantados renales la tienen de base inducida por fármacos. La detección precoz de la enfermedad en este caso sería determinante para la evolución favorable de los pacientes ${ }^{7}$.

\section{Presentación del caso}

Varón de 50 que el día 18 de marzo de 2020 acude a urgencias. Como antecedentes personales relevantes presenta enfermedad renal crónica no filiada, trasplantado renal en 2016 con creatinina 2,6-2,9 mg/dl basal e HTA. Acudió a urgencias por fiebre de $38^{\circ} \mathrm{C}$ de tres días de evolución, tos, rinorrea y diarrea con pérdida de $5 \mathrm{~kg}$ de peso. Refiere disuria desde el día previo a su ingreso en urgencias y leve molestia en injerto renal en fosa iliaca derecha sin disminución del ritmo de diuresis.

En el examen radiológico se objetivan tenues infiltrados pulmonares en el campo medio del hemitórax derecho, compatibles con la sospecha de focos neumónicos de pequeño tamaño. Se solicita PCR de Influenza $A, B$, VRS y SARS-CoV-2 y antigenuria de neumococo y legionella. Se extrae hemocultivos y urocultivos y se inicia antibioterapia empírica con ceftriaxona. No datos de infección de orina.

Al día siguiente ingresa en nuestra unidad de trasplante renal del servicio de nefrología por COVID-19, tras PCR positiva para SARS-CoV-2. EI paciente es portador de una vía venosa periférica en el miembro superior derecho, por la cual se le está administrando sueroterapia.

En contexto de infección respiratoria y diarrea presenta deterioro de función renal por hipoperfusión renal hasta creatinina de $3,9 \mathrm{mg} / \mathrm{dL}$ con urea $122, \mathrm{Na}$ en orina 33, Cloro 21 . Ingresa con una pauta de Tacrólimus $3 \mathrm{mg}$ y Everolimus 1.5 ambos en desayuno y cena. Debido al empeoramiento clínico y al tratamiento empleado para el SARS-CoV-2 (2 dosis de Tocilizumab), esta se disminuyó paulatinamente retirando el Everolimus por completo en el primer día de ingreso y el Tacrólimus el quinto. 


\section{Valoración al ingreso}

La valoración inicial se realizó siguiendo los Patrones Funcionales de Marjory Gordon (Tabla 1). Se eligió esta teoría ya que cada patrón funcional está influido por factores biológicos, culturales, sociales y espirituales, existe una expresión de integración psicosocial. Permite identificar e integrar condiciones de cada persona que deben dirigirse a mantener el equilibrio para

Tabla 1. Valoración de enfermería al ingreso según los Patrones Funcionales de Marjory Gordon

\section{PATRÓN I: Percepción y manejo de salud}

Enfermedades previas: Diverticulitis aguda, Queratosis seborreica, ERCT portador de primer trasplante renal 8 mayo 2016, hipertensión arterial, hipotiroidismo.

Antecedentes quirúrgicos: Estenosis pieloureteral izquierda. Vacunas: completa.

Toma su medicación de la forma prescrita siempre.

Prácticas de salud: Dieta equilibrada, ejercicio, revisiones médicas.

Interés ante el régimen terapéutico.

\section{VALORACIÓN RIESGO DE CAÍDAS: 1}

\section{PATRÓN II: Nutricional metabólico}

Talla: $180 \mathrm{~cm}$.

Peso $78 \mathrm{~kg}$.

IMC: 24.074

RESULTADO MUST Escala nutricional: 3 (Alto riesgo de desnutrición).

RESULTADO SNAQ Escala nutricional: 3 (Alto riesgo de desnutrición).

RESULTADO NRS 2002 Escala nutricional: 2.

RESULTADO MNA SF Escala nutricional: 8 (Riesgo de desnutrición).

No toma ningún suplemento dietético ni presenta dificultades para la masticación.

No presenta náuseas ni vómitos.

Refiere que no tiene mucho apetito.

Piel y membrana hidratada y bien perfundida.

Temperatura: $39.1^{\circ} \mathrm{C}$

\section{VALORACIÓN ESCALA DE NORTON: 17}

Portador de una FAVI en MSI

Se canaliza VVP en MSD.

\section{PATRÓN III: Eliminación}

Doble continente.

Diuresis $1700 \mathrm{cc}$ aproximadamente.

Frecuencia intestinal con diarreas, pérdida de $5 \mathrm{~kg}$ de peso en los últimos días.

No tiene estomas.

\section{PATRÓN IV: Actividad ejercicio}

Saturación de $97 \%$ con GN a 3 litros.

Presenta disnea y esfuerzo respiratorio.

TA: $148 / 78$.

Frecuencia cardiaca en rango $91 \mathrm{lpm}$.

Refiere estar cansado, puede moverse sin problemas e ir al baño, pero refiere que se encuentra "flojo".

IABVD.

\section{PATRóN V: Descanso sueño}

No alterado.

Refiere dormir las horas necesarias y no levantarse cansado.

No precisa de elementos que le ayuden a dormir.

\section{PATRÓN VI: Cognitivo perceptual}

Consciente y orientado.

No presenta alteraciones en la comunicación.

No presenta deterioros sensoriales ni cognitivos.

No refiere dolor (EVA 0 ).

Precisa de información específica (educación sanitaria).

\section{PATRÓN VII: Autoconcepto y autoestima}

Nervioso, intranquilo y preocupado por la situación en la que está.

\section{PATRÓN VIII: Rol-relaciones}

Sistema de apoyo-su familia.

\section{PATRÓN IX: Sexualidad-reproducción}

No alterado.

\section{PATRÓN X: Adaptación y tolerancia al estrés}

No alterado.

\section{PATRÓN XI: Valores y creencias}

No alterado.

así recuperar o conservar la salud. Los datos fueron recogidos mediante una entrevista personal. Se llevó a cabo la exploración física y la toma de constantes.

\section{Plan de cuidados}

En base a los datos obtenidos en la valoración, se elaboró un plan de cuidados utilizando la taxonomía NANDANOC-NIC (Tabla 2). 
Tabla 2. Diagnósticos (NANDA), resultados (NOC) e intervenciones (NIC).

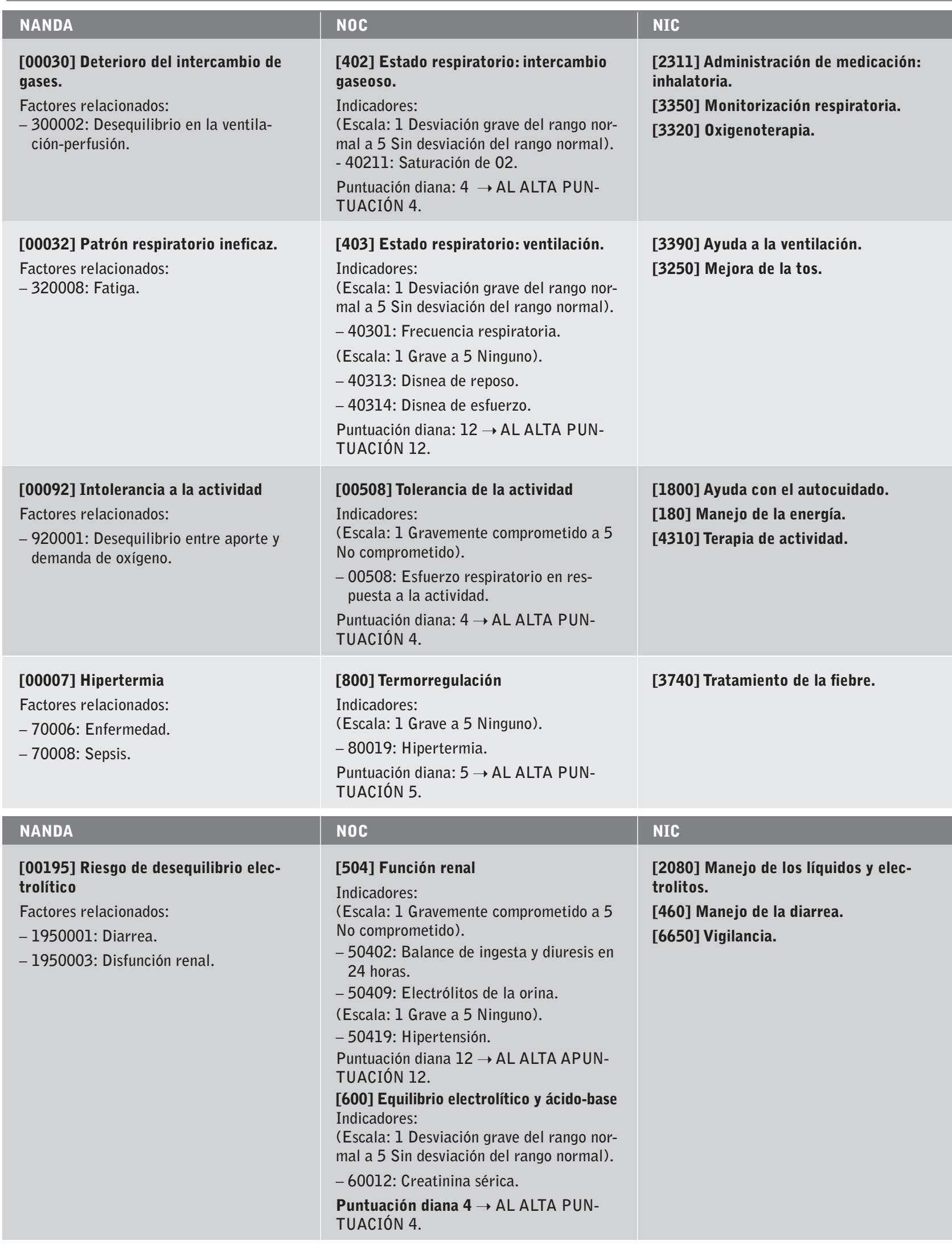




\begin{tabular}{|c|c|c|}
\hline NANDA & NOC & NIC \\
\hline $\begin{array}{l}\text { [00043] Protección ineficaz } \\
\text { Factores relacionados: } \\
\text { - 430002: Agentes farmacológicos. } \\
\text { - 430006: Perfil hematológico anormal. }\end{array}$ & $\begin{array}{l}\text { [702] Estado inmune } \\
\text { Indicadores: } \\
\text { (Escala: } 1 \text { Gravemente comprometido a } 5 \\
\text { No comprometido). } \\
\text { - 70207: Temperatura corporal. } \\
\text { - 70201: Detección de infección. } \\
\text { Puntuación diana } 8 \rightarrow \text { AL ALTA PUN- } \\
\text { TUACIÓN } 8 .\end{array}$ & $\begin{array}{l}\text { [6540] Control de infecciones. } \\
\text { [6550] Protección contra las infeccio- } \\
\text { nes. }\end{array}$ \\
\hline $\begin{array}{l}\text { [00203] Riesgo de perfusión renal } \\
\text { ineficaz } \\
\text { Factores relacionados: } \\
\text { - 2030008: Enfermedad renal. } \\
\text { - 2030016: Infección. }\end{array}$ & $\begin{array}{l}\text { [404] Perfusión tisular } \\
\text { Indicadores: } \\
\text { (Escala:1 Desviación grave del rango nor- } \\
\text { mal a } 5 \text { Sin desviación del rango normal). } \\
\text { - 40402: Diuresis. } \\
\text { - 40420: Creatinina plasmática. } \\
\text { - 40427: Equilibrio de líquidos alterado. } \\
\text { Puntuación diana } 12 \rightarrow \text { AL ALTA PUN- } \\
\text { TUACIÓN } 12 \text {. }\end{array}$ & $\begin{array}{l}\text { [4120] Manejo de líquidos. } \\
\text { [6680] Monitorización de los signos } \\
\text { vitales. }\end{array}$ \\
\hline NANDA & NOC & NIC \\
\hline $\begin{array}{l}\text { [00155] Riesgo de caídas } \\
\text { Factores relacionados: } \\
\text { - 1550014: Enfermedad aguda. }\end{array}$ & $\begin{array}{l}\text { [1912]Caídas } \\
\text { Indicadores: } \\
\text { (Escala: } 1 \text { Mayor de } 10 \text { a } 5 \text { Ninguno). } \\
\text { - 191201: Caídas en bipedestación. } \\
\text { - 191204: Caídas de la cama. } \\
\text { Puntuación diana } 10 \rightarrow \text { AL ALTA PUN- } \\
\text { TUACIÓN 10. } \\
\text { [1828] Conocimiento: prevención de } \\
\text { caídas } \\
\text { Indicadores: } \\
\text { (Escala: } 1 \text { Ningún conocimiento a } 5 \text { Cono- } \\
\text { cimiento extenso). } \\
\text { - 182802: Uso correcto de los mecanis- } \\
\text { mos de seguridad. } \\
\text { - 182803: Calzado adecuado. } \\
\text { Puntuación diana } 8 \rightarrow \text { AL ALTA PUN- } \\
\text { TUACIÓN } 8 .\end{array}$ & $\begin{array}{l}\text { [6486] Manejo ambiental: seguridad. } \\
\text { [6490] Prevención de caídas. }\end{array}$ \\
\hline $\begin{array}{l}\text { [00146] Ansiedad } \\
\text { Factores relacionados: } \\
\text { - 1460003: Amenaza de muerte. } \\
\text { - 1460007: Contagio interpersonal. } \\
\text { - 1460011: Factores estresantes. } \\
\text { - 1460012: Grandes cambios (p.ej., esta- } \\
\text { tus económico, entorno, estado de salud, } \\
\text { rol, consideración social del rol). }\end{array}$ & $\begin{array}{l}\text { [1211] Nivel de ansiedad } \\
\text { Indicadores: } \\
\text { (Escala: } 1 \text { Grave a } 5 \text { Ninguno). } \\
\text { - 121105: Inquietud. } \\
\text { - 121117: Ansiedad verbalizada. } \\
\text { - 1211129: Trastorno del sueño. } \\
\text { Puntuación diana } 12 \rightarrow \text { AL ALTA PUN- } \\
\text { TUACIÓN } 12 .\end{array}$ & $\begin{array}{l}\text { [5820] Disminución de la ansiedad. } \\
\text { [5230] Mejorar el afrontamiento. } \\
\text { [1850] Mejorar el sueño. }\end{array}$ \\
\hline
\end{tabular}




\begin{tabular}{|c|c|c|}
\hline NANDA & NOC & NIC \\
\hline $\begin{array}{l}\text { [00002] Desequilibrio nutricional: } \\
\text { ingesta inferior a las necesidades } \\
\text { Factores relacionados: } \\
\text {-20003: Incapacidad para absorber los } \\
\text { nutrientes. }\end{array}$ & $\begin{array}{l}\text { [1004] Estado nutricional } \\
\text { Indicadores: } \\
\text { (Escala: } 1 \text { Desviación grave del rango nor- } \\
\text { mal a } 5 \text { Sin desviación del rango normal). } \\
\text { - 100401: Ingestión de nutrientes. } \\
\text { - 100403: Energía. } \\
\text { Puntuación diana } 8 \rightarrow \text { AL ALTA PUN- } \\
\text { TUACIÓN } 8 . \\
\text { [101] Apetito } \\
\text { Indicadores: } \\
\text { (Escala: } 1 \text { Gravemente comprometido a } 5 \\
\text { No comprometido). } \\
\text { - 101406: Ingesta de alimentos. } \\
\text { Puntuación diana } 4 \rightarrow \text { AL ALTA PUN- } \\
\text { TUACIÓN } 4 .\end{array}$ & $\begin{array}{l}\text { [180] Manejo de la energía. } \\
\text { [1160] Monitorización nutricional. }\end{array}$ \\
\hline $\begin{array}{l}\text { [00161] Disposición para mejorar los } \\
\text { conocimientos }\end{array}$ & $\begin{array}{l}\text { [1803] Conocimiento: proceso de la } \\
\text { enfermedad } \\
\text { Indicadores: } \\
\text { (Escala: } 1 \text { Ningún conocimiento a } 5 \text { Cono- } \\
\text { cimiento extenso). } \\
\text { - 180302: Características de la enfer- } \\
\text { medad. } \\
\text { Puntuación diana } 5 \rightarrow \text { AL ALTA PUN- } \\
\text { TUACIÓN } 5 . \\
\text { [1813] Conocimiento: régimen tera- } \\
\text { péutico } \\
\text { Indicadores: } \\
\text { (Escala: } 1 \text { Ningún conocimiento a } 5 \text { Cono- } \\
\text { cimiento extenso). } \\
\text { - 181306: Régimen de medicación. } \\
\text { - 181307: Actividad física prescrita. } \\
\text { Puntuación diana } 10 \rightarrow \text { AL ALTA PUN- } \\
\text { TUACIÓN } 10 .\end{array}$ & $\begin{array}{l}\text { [5618] Enseñanza: procedimiento/tra- } \\
\text { tamiento. } \\
\text { [5602] Enseñanza: proceso de enfer- } \\
\text { medad. }\end{array}$ \\
\hline
\end{tabular}

Se dividió el plan de cuidados en función de la patología que presentaba el paciente, dando prioridad a aquellos que debían de tratarse de forma más urgente.

Debido a la retirada de inmunosupresores se detectó una complicación potencial añadida a los diagnósticos de enfermería ya nombrados anteriormente, rechazo agudo del trasplante renal.

\section{Evaluación del plan}

En los diagnósticos de Patrón respiratorio ineficaz y en Deterioro del intercambio de gases inicialmente la evolución fue mala precisando inhaladores y pasando de oxigenoterapia con gafas nasales a 3 litros por minuto $(\mathrm{lpm})$ a mascarilla reservorio a 10 litros para mantener saturaciones superiores a $95 \%$. Llegando incluso en días posteriores a precisar flujos de oxígeno con mascarilla reservorio de $20 \mathrm{lpm}$. A partir del séptimo día y tras realizar cambios posturales colocando al paciente en posición prono para mejorar la ventilación se consiguió disminuir el aporte de oxígeno paulatinamente hasta el noveno día en el que el paciente mantiene saturaciones por encima de $95 \%$ con GN a 3 litros. Tras 12 días se consiguió una saturación mayor de $95 \%$ en basal.

Para llevar a cabo la terapia de actividad una vez que el paciente se encontró respiratoriamente estable se fomentó la movilización precoz activa. Se le animó a sentarse y a realizar ejercicios sencillos y actividades de la vida diaria con el fin de recuperar su indepen- 
dencia en el autocuidado. Conforme avanzaba el ingreso caminó por la habitación sin presentar taquicardia, desaturación, ni trabajo respiratorio. A la hora de realizar la actividad física se acompañó para prevenir caídas y realizar un adecuado manejo ambiental.

La fiebre y posterior febrícula mantenida en los primeros días se controló gracias a la administración de antitérmicos habituales y aplicación de medidas físicas, desapareciendo tras cinco días.

En lo referente a la función renal del injerto y a la inmunosupresión (IS), se disminuyeron los inmunosupresores, por lo que el riesgo de rechazo agudo del trasplante fue mayor. Los niveles plasmáticos se controlaron cada 48 horas. Una vez superada la fase aguda y con la función renal mantenida, se fue reintroduciendo la IS a dosis bajas comenzando únicamente con el Tacrólimus.

En relación al estado emocional, se acompañó al paciente en todo momento, se realizó escucha activa y se resolvieron todas las dudas que pudiera tener sobre su situación. Se le ayudó a controlar la ansiedad mediante estrategias de relajación y autocontrol, y a manejar el afrontamiento consiguiendo que se sintiese más tranquilo conforme mejoraba su salud. Debido al contexto excepcional en el que nos encontrábamos no se permitían visitas en el hospital, en algunas ocasiones nos contaba que se sentía solo y que estaba preocupado por su familia por lo que se realizaron videollamadas para que se pudieran comunicar y así disminuir la ansiedad provocada por esta situación.

Se realizó educación sanitaria incidiendo en la importancia de la actividad progresiva y la administración de medicación. El paciente verbalizó en todo momento entender lo que se le explicaba y ser capaz de realizarlo sin incidencias.

Respecto al patrón nutricional metabólico, el paciente llegó a urgencias con $5 \mathrm{~kg}$ de pérdida de peso debido a astenia y diarrea manteniéndose estas los primeros días de ingreso. Al alta se consiguió una ganancia de peso de $2 \mathrm{~kg}$.

\section{Discusión}

La infección COVID-19 podría comportarse de forma diferente en pacientes trasplantados ${ }^{10}$, presentando un curso más severo de la misma ${ }^{11}$. El tratamiento inmunosupresor utilizado en pacientes trasplantados podría ser determinante para favorecer una infección, aunque a su vez podría atenuar la respuesta inflamatoria provocada por la COVID-197,8,9. El manejo de la retirada del tratamiento inmunosupresor debe ser individualizado para cada paciente. En nuestro caso y siguiendo las recomendaciones en el manejo de la pandemia por coronavirus SARS-CoV-2 (COVID-19) en pacientes con trasplante renal ${ }^{7}$, se retiraron los inmunosupresores del paciente durante su estancia hospitalaria, y fue una vez dado de alta y en la consulta ambulatoria de Trasplante Renal donde se reinició su pauta habitual de inmunosupresión. En un principio, se temía por la pérdida del injerto debido a la retirada de la medicación inmunosupresora. Sin embargo, la evolución durante el ingreso hospitalario fue óptima, y una vez dado de alta, se reinició el tratamiento consiguiendo valores de la función renal similares a los previos de la infección por COVID-19.

Según el último informe elaborado por la Organización Nacional de Trasplantes para pacientes trasplantados con COVID-1912, recopila que el manejo de la inmunosupresión en estos pacientes fue heterogéneo en función del tipo de trasplante. De los 466 pacientes registrados, a un $85 \%$ de los mismos se llevó a cabo un ajuste del tratamiento inmunosupresor, optándose por disminuir la dosis o suspender la administración de diferentes inmunosupresores. Aunque se lograron los objetivos planteados en cuanto a los diagnósticos relacionados con el estado respiratorio del paciente, se hizo hincapié en la importancia de que una vez dado de alta el paciente continuara realizando entrenamiento aeróbico y de fuerza de manera progresiva y continuara llevando a cabo las técnicas de drenaje de secreciones para mejorar la ventilación y evitar así un posible reingreso. Aspectos que están en consonancia con las recomendaciones del área de fisioterapia respiratoria de la SEPAR ${ }^{13}$ y las directrices dadas por el equipo de rehabilitación de nuestro hospital.

La ansiedad provocada por la incertidumbre de la enfermedad y el aislamiento al que el paciente es sometido, son aspectos importantes en el cuidado de estas personas. En el documento recomendaciones para el manejo del paciente con enfermedad renal crónica ante el SARS-CoV-2, se resalta la importancia de asegurar el bienestar psicosocial del paciente renal. Es vital promover la escucha activa, destacando la información al paciente y familiares como un pilar fundamental en la atención clínica. Además, implementando nuevas acciones para favorecer las comunicaciones entre ellos durante el aislamiento, como fue el uso de las llamadas telefónicas y las videollamadas. 
El paciente trasplantado renal presenta una alta vulnerabilidad especial frente al SARS-CoV-2 debido a su fragilidad intrínseca, comorbilidad añadida y la utilización de un tratamiento inmunosupresor. En nuestro caso el paciente se fue de alta con una función renal similar a la previa y con la inmunosupresión reintroducida, logrando los objetivos planteados al inicio y dejando patente que el papel de la enfermera renal ha sido fundamental durante todo el proceso de recuperación y afrontamiento de la enfermedad.

A pesar de todo, aún queda mucho recorrido para conocer en profundidad todo lo que implica la infección por SARSCoV-2 en pacientes trasplantados. Es importante continuar investigando sobre la COVID-19 en pacientes nefrológicos, contando con planes de cuidados nefrológicos específicos para así favorecer la continuidad de cuidados.

Recibido: 05-08-2020

Revisado: 10-08-2020

Modificado: 26-08-2020

Aceptado: 30-08-2020

\section{Bibliografía}

1. Coronavirus disease (COVID-19) pandemic. World Health Organization. [Consultado 3 jun 2020]. Disponible en: https://www.who.int/emergencies/diseases/ novel-coronavirus-2019.

2. Ministerio de Sanidad. Actualización $n^{\circ}$ 177. Enfermedad por el coronavirus (COVID-19). [Consultado 4 Ago 2020]. Disponible en: https://www.mscbs.gob. es/en/profesionales/saludPublica/ccayes/alertasActual/nCov-China/documentos/Actualizacion_177_COVID-19.pdf.

3. Instituto de Salud Carlos III.Vigilancia de los excesos de mortalidad por todas las causas: MoMo. 19 Julio 2020 [Consultado 5 ago 2020]. Disponible en:https:// www.isciii.es/QueHacemos/Servicios/VigilanciaSaIudPublicaRENAVE/EnfermedadesTransmisibles/ MoMo/Documents/informesMoMo2020/MoMo_Situacion\%20a\%2019\%20de\%20julio_CNE.pdf.
4. Houlshue $M L$, DeBolt $C$, Lindquist $S$, Lofy $K H_{\text {, }}$ Wiesman J, Bruce H et al. First Case of 2019 Novel Coronavirus in the United States. N Engl J Med. 2020;382(10):929-36.

5. Wang D, Hu B, Hu C, Zhu F, Liu X, Zhang J et al. Clinical Characteristics of 138 Hospitalized Patients With 2019 Novel Coronavirus-Infected Pneumonia in Wuhan, China. JAMA. 2020;323(11):1061-9.

6. Mo $P$, Xing $Y$, Xiao $Y$, Deng $L$, Zhao Q, Wang $H$ et al. Clinical characteristics of refractory COVID-19 pneumonia in Wuhan, China. Clin Infect Dis. 2020 Mar 16:ciaa270. [En prensa].

7. López V, Vázquez T, Alonso-Titos J, Cabello M, Alonso $A$, Beneyto I et al. Recomendaciones en el manejo de la pandemia por coronavirus SARS-Cov-2 (Covid-19) en paciente con trasplante renal. Nefrología 2020;40(3):265-71.

8. Sequera Ortiz $P$, Quiroga $B$, Arriba de la Fuente $G$, Macía M, Salgueira M, Pino MD. Protocolo de actuación ante la epidemia de enfermedad por coronavirus en los pacientes de diálisis y trasplantados renales. Nefrología 2020;40(3):223-7.

9. Naicker S, Yang C, Hwang S, Liu B, Chen J, Jha V. The Novel Coronavirus 2019 epidemic and kidneys. Kidney Int. 2020 May;97(5):824-8.

10. Albas Gómez-Beltrán P, Domínguez Carranza E, Medero Rubio F, Pérez Márquez M. Recomendaciones para el manejo del paciente con enfermedad renal crónica ante el SARS-CoV-2. Enferm Clin. 2020 May [En prensa].

11. Fernández-Ruiz, Andrés A, Loinaz C, Delgado JF, López-Medrano F, San Juan R et al. COVID-19 in solid organ trasplant recipients: A single-center case series from Spain. Am J Transplant. 2020 Jul;20(7):1849-58.

12. Coll E, Gayoso J, Martínez JR, Domínguez-Gil B. Informe casos Covid-19 en pacientes trasplantado. Organización Nacional de Trasplantes. 18 Mayo 2020. [Consultado 6 Ago 2020]. Disponible en: https://www.seden.org/files/news/INFORME\%20 CASOS\%20COVID\%2019\%20EN\%20PACIENTES\%20TRASPLANTADOS\%20\%2018\%20 MAY0_b5f4.pdf. 
13. Arbilaga A, Pardás M, Escudero R, Rodriguez R, Alcaraz $\mathrm{V}$, Llanes $\mathrm{S}$ et al. Fisioterapia respiratoria en el manejo del paciente con COVID-19: Recomendaciones general. Área de fisioterpia respiratoria. Sociedad Española de Neumología y Cirugía Torácica. SEPAR. 26 Marzo 2020. [Consultado 5 Ago 2020]. Disponible en: http://svmefr.com/wp-content/ uploads/2020/03/COVID19-SEPAR-26_03_20.pdf.

Este artículo se distribuye bajo una Licencia Creative Commons Atribución-NoComercial 4.0 Internacional. https://creativecommons.org/licenses/by-nc/4.0/

\section{Open Access (c) ()}

